\title{
Kidney Blood Pressure Research
}

\section{Frailty in Chinese Peritoneal Dialysis Patients: Prevalence and Prognostic Significance}

\author{
Jack Kit-Chung Ng Bonnie Ching-Ha Kwan Kai-Ming Chow \\ Phyllis Mei-Shan Cheng Man-Ching Law Wing-Fai Pang Chi-Bon Leung \\ Philip Kam-Tao Li Cheuk-Chun Szeto
}

Carol \& Richard Yu Peritoneal Dialysis Research Centre, Department of Medicine \& Therapeutics,

Prince of Wales Hospital, The Chinese University of Hong Kong, Shatin, Hong Kong, China

\section{Key Words}

Peritoneal dialysis • Nutrition • Cardiovascular disease

\begin{abstract}
Background/Aims: Previous studies showed that frailty is prevalent in both pre-dialysis and dialysis patients. However, the prevalence and prognostic implication of frailty in Chinese peritoneal dialysis (PD) patients remain unknown. Methods: We used a validated questionnaire to determine the Frailty Score of 193 unselected prevalent PD patients. All patients were then followed for 2 years for their need of hospitalization and mortality. Results: Amongst the 193 patients, 134 (69.4\%) met the criteria of being frail. Frailty Score significantly correlated with Charlson's comorbidity score $(r=0.40, p<0.0001)$, Malnutrition Inflammation Score $(r=0.59, p<0.0001)$, and inversely with Subjective Global Assessment score $(r=-0.44, p$ $<0.0001)$. Frailty was closely associated with the need of hospitalization. Patients with nil, mild, moderate, and severe frailty required $2.4 \pm 6.0,1.6 \pm 1.6,2.7 \pm 2.5,5.2 \pm 4.8$ hospital admissions per year, respectively $(p<0.0001)$, and they stayed in hospital for $6.4 \pm 9.2,5.3 \pm$ $6.2,10.0 \pm 10.4,12.9 \pm 20.1$ days per hospital admission, respectively $(p<0.0001)$. However, Frailty Score was not an independent predictor of patient or technique survival. Conclusions: Frailty is prevalent among Chinese PD patients. Frail PD patients have a high risk of requiring hospitalization and their hospital stay tends to be prolonged. Early identification may allow timely intervention to prevent adverse health outcomes in this group of patients
\end{abstract}

(C) 2016 The Author(s)

Published by S. Karger AG, Basel

\section{Introduction}

First described by geriatricians, frailty is a state of increase in vulnerability to stressors owing to decrease in reserve or dysregulation in multiple physiological systems [1]. This 


\section{Kidney Blood Pressure Research}

limited physiological reserve predisposes individuals to disproportionate change in health status albeit an apparent minor insult, which results in fall, delirium and disability [2].

Frailty is common amongst chronic kidney disease (CKD) patients. A cross sectional study evaluated frailty in elderly patients with CKD (Cardiovascular Health Study cohort) by the original Fried's criteria and reported a prevalence of 15\% [3]. On the other hand, Johansen et al described a cohort of 2275 incident dialysis patients and concluded that $67 \%$ of them were frail by a modified Fried's criteria [4]. However, study on the prevalence of frailty in Chinese CKD or dialysis patients is scarce.

Frail patients with CKD or on dialysis shared similar adverse clinical consequences as frail general medical patients. A national cohort consisting of 10256 Americans revealed a two-fold increase in risk of death in frail adults with CKD compared with those who were not frail [5]. Johansen et al also demonstrated that frailty in dialysis population independently predicted mortality and hospitalization after adjustment for age, gender, dialysis modalities and comorbidities [4]. In this study, we determined the prevalence of frailty in Chinese peritoneal dialysis (PD) patients. We also explored the relation between frailty and clinical characteristics, comorbid conditions, nutritional status, as well as the association between frailty and hospitalizations, peritonitis and survival in Chinese PD patients.

\section{Patients and Methods}

\section{Study population}

Amongst a total of 397 Chinese PD patients in our unit, we invited 196 consecutive patients who visited the dialysis clinic of our hospital from April to May 2012; 3 declined to participate. This study was approved by the Joint Chinese University Hong Kong-New Territories East Cluster Clinical Research Ethics Committee.

\section{Overall arrangement}

After informed consent, frailty was assessed by a standard questionnaire, and a nutritional assessment was performed at the same time. Baseline clinical and laboratory data were obtained by chart review. The modified Charlson's Comorbidity Index was calculated by methods previously described [6]. This Index encompassed a variety of common comorbidities (including coronary artery disease, liver disease, malignancy), each of which was assigned a score from 1 to 6 . The summation of score was shown to predict both patient and technique survivals in PD patients respectively [6].

\section{Assessment of frailty}

We used a validated Chinese questionnaire which consisted of 30 dichotomous questions [7]. The Frailty Score (FQ) was computed, with a range 0 to 30. The degree of frailty was then classified into 'none' (FQ $\leq 5)$, 'mild' (FQ 6 to 8), 'moderate' (FQ 9 to 11) and 'severe' (FQ 12 or above).

\section{Dialysis adequacy and nutritional assessment}

Dialysis adequacy was measured by Kt/V and residual glomerular filtrate rate (GFR) by 24-hour dialysate and urine collection. Nutritional status was represented by serum albumin level, subjective global assessment (SGA) [8], comprehensive malnutrition-inflammation score (MIS) [9], normalized protein nitrogen appearance (nPNA) [10], and fat-free edema-free body mass (FEBM) [11].

\section{Bioimpedance spectroscopy study}

We used the multi-frequency bioimpedance spectroscopy (Body Composition Monitor, Fresenius Medical Care, Germany) as described previously [12]. The test was generally performed in the afternoon during PD fluid dwell. We computed the lean tissue mass (LTM), adipose tissue mass (ATM), and volume of over-hydration.

Follow-up and outcome measures

The planned duration of follow up was 2 years. Primary outcomes included the number of hospital 


\section{Kidney Blood Pressure Research}

admission for all causes, number of hospital admission related to cardiovascular events, total length of hospital stay, and patient survival. Secondary outcomes included peritonitis rate and technique survival. Cardiovascular events were defined as acute coronary syndrome, hospitalization for heart failure, peripheral vascular disease or stroke. Peritonitis rate was calculated by dividing the number of peritonitis episodes by duration of follow-up, and expressed as episodes per year. Patient death, transfer to long term hemodialysis, and kidney transplantation are considered as events for the analysis of technique survival.

\section{Statistical analysis}

Statistical analysis was performed by SPSS for Windows software version 17.0 (SPSS Inc., Chicago). The planned sample size was 200, which was based on experience with previous reports [3,4], which suggested that 200 patients would be sufficient for a reasonable dermination of prevalence and risk factors of frailty. Descriptive data was presented as mean \pm SD if normally distributed, or median (inter-quartile range) otherwise. Patients were grouped according to the degree of frailty as defined above for analysis. Clinical parameters were compared by Student's t-test or one way analysis of variance (ANOVA) for continuous variables, and Chi-square test for categorical variables. Correlation between continuous variables was explored by the Pearson's or Spearman rank order correlation coefficient as appropriate.

The number of hospital admission and duration of hospitalization were compared between frailty groups after adjusted for the duration of follow up. Log transformation was performed because the data were highly skewed. The log-linear regression model was then used to analyze hospitalization. Potential confounders, including age, Charlson's comorbidity score, serum albumin, total Kt/V, NPNA and residual GFR, were added to the model. Backward stepwise elimination was used to determine the independent predictor of hospitalization.

Kaplan-Meier method was used to present the survival data, and log-rank test was used to compare between survival curves. The Cox proportional hazards model was then used to adjust for potential confounders and identify independent predictors of patient and technique survival. In addition to the degree of frailty, the Cox models were constructed by similar clinical parameters used in the analysis of hospitalization. These parameters were selected because of their reported significance in determining the prognosis of PD patients. Backward stepwise elimination was applied to remove insignificant variables. A P value of less than 0.05 was considered significant. All probabilities were two-tailed.

\section{Results}

We studied 193 patients (97 males). The mean age was $60.6 \pm 12.1$ years and the mean duration of dialysis was $50.1 \pm 47.5$ months. The distribution of age, underlying renal diagnosis, and prevalence of comorbid condition were similar to our overall dialysis population. The distribution of the Frailty Score was summarized in Figure 1. The mean Frailty Score was $8.4 \pm 4.6$. Amongst the 193 patients, 134 (69.4\%) met the criteria of being frail. The degree of frailty was classified as mild, moderate and severe in 57 (29.5\%), 33 (17.1\%), and 44 patients $(22.8 \%)$, respectively.

\section{Relation with clinical characteristics}

The demographic, baseline clinical and biochemical information were compared between patient groups with different degree of frailty and summarized in Tables 1 and 2, respectively. Frailty was more common in elderly patients, and over $75 \%$ of patients aged over 60 years old fulfilled the criteria of being frail. Nevertheless, the prevalence of frailty was $64 \%$ amongst patients younger than 50 years old. The Frailly Score had modest but significant correlation with Charlson comorbidity score (Spearman's rank correlation coefficient, $r=0.40, p<0.0001)$, MIS ( $r=0.59, p<0.0001)$, and inversely with serum albumin $(r=-0.33$, $p<0.0001)$, overall SGA score $(r=-0.44, p<0.0001)$, weekly total Kt $/ V(r=-0.26, p=0.001)$, and residual GFR $(r=-0.22, p=0.003)$. Frailty Score also correlated significantly with the volume of overhydration $(r=0.23, p=0.002)$ but inversely with LTM $(r=-0.33, p<0.0001)$. 


\section{Kidney Blood Pressure Research}

Relation hospitalization

The patients were followed for $22.7 \pm 7.0$ months. During this period, there were 788 hospital admissions for a total of 6780 days; $12.4 \%$ of patients did not require any hospitalization during the study period. The need of hospitalization between different severity of frailty is compared and summarized in Table 3 . In short, the more frail the patient, the longer the total duration of hospitalization during the study period. The number of hospital admission, for

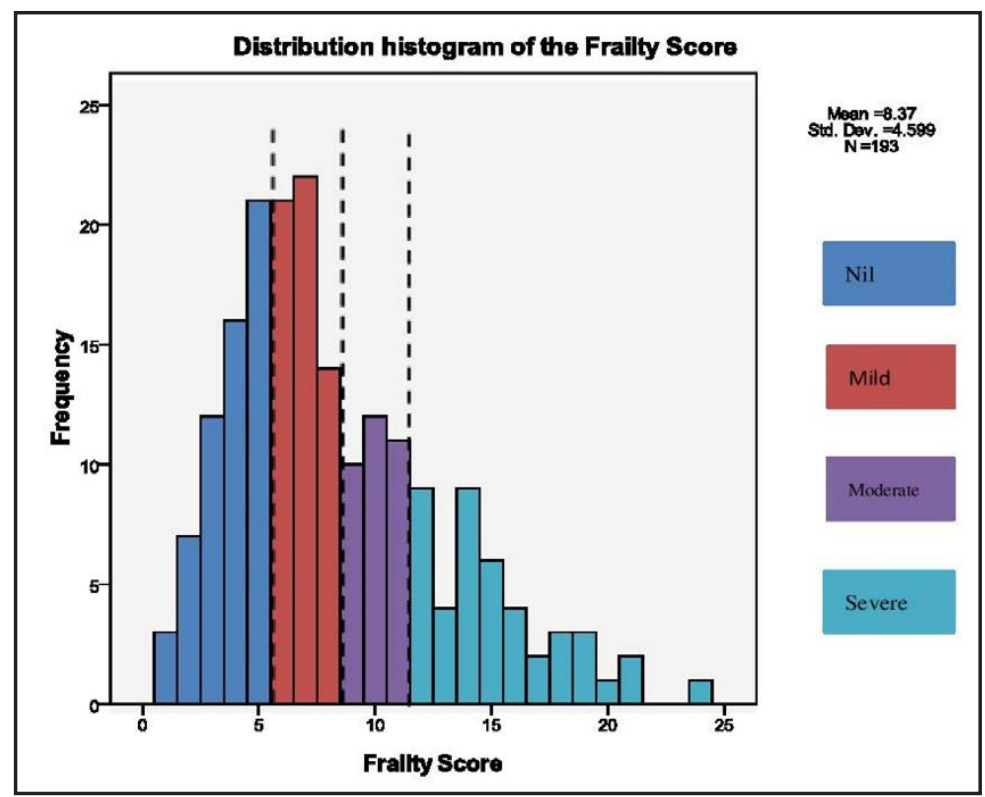

Fig. 1. Distribution histogram of the Frailty score.

Table 1. Baseline demographic and clinical data

\begin{tabular}{|c|c|c|c|c|c|}
\hline Frailty & nil & mild & moderate & severe & P value \\
\hline Frailty score & $\leq 5$ & $6-8$ & $9-11$ & $\geq 12$ & \\
\hline No. of patients & 59 & 57 & 33 & 44 & \\
\hline Male sex (\%) & $35(59.3 \%)$ & $27(47.4 \%)$ & $13(39.4 \%)$ & $22(50.0 \%)$ & 0.30 \\
\hline Age (year) & $56.6 \pm 11.5$ & $58.3 \pm 11.7$ & $60.0 \pm 10.7$ & $69.4 \pm 9.8$ & $<0.0001$ \\
\hline Duration of dialysis (month) & $45.8 \pm 48.5$ & $46.6 \pm 47.0$ & $48.9 \pm 51.1$ & $61.5 \pm 43.8$ & 0.01 \\
\hline Body height $(\mathrm{cm})$ & $160.6 \pm 11.2$ & $160.0 \pm 7.8$ & $157.2 \pm 11.8$ & $158.6 \pm 8.4$ & 0.11 \\
\hline Body weight (kg) & $63.6 \pm 13.8$ & $64.2 \pm 12.8$ & $63.4 \pm 16.2$ & $61.7 \pm 12.6$ & 0.42 \\
\hline Body mass index $\left(\mathrm{kg} / \mathrm{m}^{2}\right)$ & $24.7 \pm 4.9$ & $25.1 \pm 4.8$ & $25.7 \pm 5.7$ & $24.4 \pm 3.6$ & 0.94 \\
\hline \multicolumn{6}{|l|}{ Blood pressure (mmHg) } \\
\hline Systolic & $144.2 \pm 22.7$ & $141.5 \pm 19.7$ & $144.7 \pm 13.6$ & $142.2 \pm 27.2$ & 0.87 \\
\hline Diastolic & $82.5 \pm 12.5$ & $80.5 \pm 11.0$ & $77.8 \pm 12.8$ & $72.7 \pm 12.0$ & $<0.0001$ \\
\hline Renal diagnosis, no. of cases (\%) & & & & & 0.001 \\
\hline Glomerulonephritis & $23(39.0 \%)$ & $23(40.4 \%)$ & $11(33.3 \%)$ & $4(9.1 \%)$ & \\
\hline Diabetic nephropathy & $11(18.6 \%)$ & $17(29.8 \%)$ & $16(48.5 \%)$ & $23(52.3 \%)$ & \\
\hline Polycystic kidney & $3(5.1 \%)$ & $7(12.3 \%)$ & $1(3.0 \%)$ & $0(0 \%)$ & \\
\hline Hypertensive nephrosclerosis & $4(6.8 \%)$ & $4(7.0 \%)$ & $1(3.0 \%)$ & $8(18.2 \%)$ & \\
\hline Obstructive uropathy & $6(10.2 \%)$ & $1(1.8 \%)$ & $1(3.0 \%)$ & $3(6.8 \%)$ & \\
\hline Others / unknown & $12(20.3 \%)$ & $5(8.8 \%)$ & $2(6.0 \%)$ & $6(13.6 \%)$ & \\
\hline \multicolumn{6}{|c|}{ Pre-existing vascular disease, no. of cases (\%) } \\
\hline Diabetes & $16(27.1 \%)$ & $20(35.1 \%)$ & $16(48.5 \%)$ & $28(63.6 \%)$ & $<0.0001$ \\
\hline Coronary heart disease & $3(5.1 \%)$ & $5(8.8 \%)$ & $3(9.1 \%)$ & $12(27.3 \%)$ & 0.002 \\
\hline Cerebrovascular disease & $4(6.8 \%)$ & $7(12.3 \%)$ & $3(9.1 \%)$ & $8(18.2 \%)$ & 0.12 \\
\hline Peripheral vascular disease & $0(0 \%)$ & $1(1.8 \%)$ & $0(0 \%)$ & $6(13.6 \%)$ & 0.002 \\
\hline Charlson's comorbidity index & $4.3 \pm 2.0$ & $4.6 \pm 2.0$ & $5.3 \pm 2.2$ & $6.6 \pm 2.0$ & $<0.0001$ \\
\hline
\end{tabular}

Data are presented as mean \pm standard deviation. Spearman rank order correlation was used to compare continuous variables whereas Chi-Square test was used to compare categorical variables.

all causes as well as for cardiovascular events, increased with the severity of frailty, and all patients who were severely frail had at least one hospital admission during the study period. Moreover, the duration of stay per admission was significantly longer in patients with a higher degree of frailty, and patients who were severely frail had to stay approximately two-fold longer for each admission when compared with those who were not or mildly frail (Table 3). In addition, the Frailty Score also correlated significantly with number of hospital admission for all cause ( $r=0.32, p<0.0001)$, number of hospital admission for cardiovascular events 


\section{Kidney Blood Pressure Research}

Table 2. Baseline biochemical data and dialysis prescription

\begin{tabular}{|c|c|c|c|c|c|}
\hline Frailty & nil & mild & moderate & severe & P value \\
\hline No. of patients & 59 & 57 & 33 & 44 & \\
\hline Malnutrition inflammation score & $6.0 \pm 3.1$ & $7.8 \pm 2.6$ & $9.1 \pm 3.2$ & $12.2 \pm 4.5$ & $<0.0001$ \\
\hline Subjective Global Assessment score & $5.3 \pm 0.8$ & $5.1 \pm 1.0$ & $4.7 \pm 0.7$ & $4.2 \pm 0.9$ & $<0.0001$ \\
\hline Hemoglobin $(\mathrm{g} / \mathrm{dL})$ & $9.32 \pm 1.25$ & $9.33 \pm 1.48$ & $8.72 \pm 1.31$ & $8.98 \pm 1.29$ & 0.05 \\
\hline \multicolumn{6}{|l|}{ Serum biochemistry } \\
\hline Albumin $(\mathrm{g} / \mathrm{L})$ & $36.2 \pm 7.1$ & $35.7 \pm 4.4$ & $34.7 \pm 3.9$ & $31.7 \pm 5.7$ & $<0.0001$ \\
\hline Calcium (mmol/l) & $2.32 \pm 0.14$ & $2.33 \pm 0.17$ & $2.38 \pm 0.15$ & $2.36 \pm 0.14$ & 0.10 \\
\hline Phosphate (mmol/l) & $1.71 \pm 0.39$ & $1.80 \pm 0.50$ & $1.85 \pm 0.46$ & $1.82 \pm 0.44$ & 0.12 \\
\hline Parathyroid hormone (pmol/l) & $69.0 \pm 101.1$ & $59.8 \pm 65.2$ & $70.2 \pm 74.6$ & $72.5 \pm 71.3$ & 0.30 \\
\hline \multicolumn{6}{|l|}{ Lipid profile } \\
\hline Total cholesterol (mmol/l) & $5.18 \pm 1.21$ & $4.81 \pm 1.11$ & $5.02 \pm 1.76$ & $4.48 \pm 1.26$ & 0.01 \\
\hline Triglyceride (mmol/l) & $1.67 \pm 0.94$ & $1.84 \pm 1.57$ & $2.00 \pm 1.26$ & $1.93 \pm 1.48$ & 0.32 \\
\hline LDL cholesterol (mmol/l) & $3.02 \pm 1.05$ & $2.83 \pm 0.95$ & $2.88 \pm 1.46$ & $2.51 \pm 1.04$ & 0.02 \\
\hline HDL cholesterol (mmol/l) & $1.38 \pm 0.47$ & $1.31 \pm 0.42$ & $1.21 \pm 0.54$ & $1.18 \pm 0.43$ & 0.02 \\
\hline \multicolumn{6}{|l|}{ Peritoneal transport } \\
\hline Ultrafiltration volume (L) & $0.33 \pm 0.22$ & $0.38 \pm 0.19$ & $0.33 \pm 0.24$ & $0.31 \pm 0.24$ & 0.83 \\
\hline $\mathrm{D} / \mathrm{P}$ creatinine at 4 hour & $0.66 \pm 1.33$ & $0.61 \pm 0.13$ & $0.63 \pm 0.15$ & $0.62 \pm 0.15$ & 0.19 \\
\hline MTAC creatinine $(\mathrm{ml} / \mathrm{min} / 1.73 \mathrm{~m} 2)$ & $10.11 \pm 4.66$ & $8.52 \pm 3.83$ & $9.85 \pm 5.78$ & $9.13 \pm 4.97$ & 0.28 \\
\hline \multicolumn{6}{|l|}{ Dialysis prescription } \\
\hline Assisted PD, no. of cases (\%) & $0(0 \%)$ & $7(12.3 \%)$ & $6(18.2 \%)$ & $17(38.6 \%)$ & $<0.0001$ \\
\hline Daily exchange volume (L/day) & $7.0 \pm 1.5$ & $6.8 \pm 1.4$ & $6.1 \pm 1.1$ & $6.5 \pm 1.1$ & 0.01 \\
\hline \multicolumn{6}{|l|}{ Dialysis adequacy } \\
\hline Weekly total Kt/V & $1.96 \pm 0.52$ & $2.09 \pm 0.48$ & $1.91 \pm 0.50$ & $1.74 \pm 0.44$ & 0.001 \\
\hline Residual GFR (ml/min/1.73m2) & $2.2 \pm 2.5$ & $2.4 \pm 2.6$ & $1.2 \pm 1.7$ & $0.9 \pm 1.2$ & 0.005 \\
\hline NPNA (g/kg/day) & $1.08 \pm 0.22$ & $1.16 \pm 0.24$ & $1.14 \pm 0.26$ & $1.05 \pm 0.28$ & 0.39 \\
\hline FEBM $(\%)$ & $55.8 \pm 15.7$ & $52.3 \pm 16.7$ & $56.8 \pm 14.4$ & $51.7 \pm 11.7$ & 0.26 \\
\hline \multicolumn{6}{|l|}{ Body composition } \\
\hline Overhydration (L) & $2.49 \pm 2.83$ & $3.00 \pm 3.16$ & $2.91 \pm 2.62$ & $4.19 \pm 2.32$ & 0.004 \\
\hline Lean tissue mass (\%) & $67.5 \pm 14.8$ & $62.7 \pm 12.5$ & $62.1 \pm 13.8$ & $53.6 \pm 11.3$ & $<0.0001$ \\
\hline Adipose tissue mass (\%) & $30.5 \pm 15.4$ & $35.1 \pm 13.2$ & $36.8 \pm 13.8$ & $40.2 \pm 12.2$ & 0.001 \\
\hline
\end{tabular}

Data are presented as mean \pm standard deviation. Spearman rank order correlation was used to compare continuous variables whereas Chi-Square test was used to compare categorical variables. HDL, high density lipoprotein; LDL, low density lipoprotein; D/P, dialysate-to-plasma concentration ratio of creatinine; MTAC, mass transfer area coefficient; GFR, glomerular filtration rate; NPNA, normalized protein nitrogen appearance; FEBM, fatfree edema-free body mass.

Table 3. Hospitalization of the study cohort

\begin{tabular}{lccccc}
\hline Frailty & nil & mild & moderate & severe & P value \\
\hline No. of patients & 59 & 57 & 33 & 44 & \\
Without hospitalization, no. of patient (\%) & $11(18.6 \%)$ & $10(17.5 \%)$ & $3(9.1 \%)$ & $0(0 \%)$ & 0.02 \\
\hline Number of hospital admission (per year) & & & & & \\
\hline$\quad$ all cause & $2.4 \pm 6.0$ & $1.6 \pm 1.6$ & $2.7 \pm 2.5$ & $5.2 \pm 4.8$ & $<0.0001$ \\
$\quad$ cardiovascular disease & $0.5 \pm 1.0$ & $0.2 \pm 0.5$ & $1.0 \pm 1.1$ & $1.4 \pm 1.6$ & $<0.0001$ \\
\hline Duration of hospital stay & & & & & \\
\hline$\quad$ days per year & $18.3 \pm 39.1$ & $10.2 \pm 13.4$ & $27.4 \pm 30.1$ & $58.5 \pm 72.6$ & $<0.0001$ \\
$\quad$ days per hospital admission & $6.4 \pm 9.2$ & $5.3 \pm 6.2$ & $10.0 \pm 10.4$ & $12.9 \pm 20.1$ & $<0.0001$ \\
\hline
\end{tabular}

Data are presented as mean \pm standard deviation. Spearman rank order correlation was used to compare continuous variables whereas Chi-Square test was used to compare categorical variables.

$(\mathrm{r}=0.35, \mathrm{p}<0.0001)$, and total duration of hospital stay $(\mathrm{r}=0.39, \mathrm{p}<0.0001)$. By using the log-linear regression model to adjust for potential confounders, Frailty Score remained an independent factor associated with number of hospitalizations for all causes (beta $=0.29$, $\mathrm{p}<0.0001$ ), number of hospitalizations related to cardiovascular events (beta $=0.37, p$ $<0.0001$ ), and total length of hospital stay (beta $=0.34, \mathrm{p}<0.0001$ ). 


\section{Kidney \\ Blood Pressure Research}

Table 4. Peritonitis, death and technique failure

\begin{tabular}{|c|c|c|c|c|c|}
\hline Frailty & nil & mild & moderate & severe & $P$ value \\
\hline No. of patients & 59 & 57 & 33 & 44 & \\
\hline \multicolumn{6}{|l|}{ Peritonitis } \\
\hline peritonitis-free, no. of patient (\%) & $38(64.4 \%)$ & $36(63.2 \%)$ & $23(69.7 \%)$ & $30(68.2 \%)$ & 0.9 \\
\hline peritonitis rate (episode/year) & 0.31 & 0.26 & 0.25 & 0.38 & 0.6 \\
\hline Cause of death, no. of patient (\%) & & & & & 0.4 \\
\hline \multicolumn{6}{|l|}{ Vascular diseases } \\
\hline Cardiovascular disease & $3(5.1 \%)$ & $3(5.3 \%)$ & $0(0 \%)$ & $2(4.5 \%)$ & \\
\hline Cerebrovascular disease & $2(3.4 \%)$ & $2(3.5 \%)$ & $2(6.1 \%)$ & $1(2.3 \%)$ & \\
\hline \multicolumn{6}{|l|}{ Infections } \\
\hline Peritonitis & $2(3.4 \%)$ & $1(1.8 \%)$ & $1(3.0 \%)$ & $6(13.6 \%)$ & \\
\hline Non-peritonitis infection & $1(1.7 \%)$ & $3(5.3 \%)$ & $4(12.1 \%)$ & $14(31.8 \%)$ & \\
\hline \multicolumn{6}{|l|}{ Others } \\
\hline Termination of dialysis & $0(0 \%)$ & $1(1.8 \%)$ & $2(6.1 \%)$ & $1(2.3 \%)$ & \\
\hline Malignancy & $2(3.4 \%)$ & $0(0 \%)$ & $3(9.1 \%)$ & $0(0 \%)$ & \\
\hline Miscellaneous/ unknown & $0(0 \%)$ & $1(1.8 \%)$ & $2(6 \%)$ & $2(4.5 \%)$ & \\
\hline All deaths (\%) & $10(16.9 \%)$ & $11(19.3 \%)$ & $14(42.4 \%)$ & $26(59.1 \%)$ & $<0.0001$ \\
\hline Conversion to hemodialysis, no. of patient (\%) & $3(5.1 \%)$ & $3(5.3 \%)$ & $0(0 \%)$ & $2(4.5 \%)$ & 0.6 \\
\hline Kidney transplantation, no. of patient (\%) & $6(10.2 \%)$ & $4(7.0 \%)$ & $0(0 \%)$ & $1(2.3 \%)$ & 0.15 \\
\hline Transferal to other centers, no. of patient (\%) & $0(0 \%)$ & $1(1.8 \%)$ & $0(0 \%)$ & $3(6.8 \%)$ & 0.08 \\
\hline
\end{tabular}

Relation with peritonitis and survival

During the study period, there were 109 peritonitis episodes in 66 patients; 127 patients $(65.8 \%)$ had no peritonitis. The overall peritonitis rate was 0.30 episodes per year. There was no significant relation between peritonitis rate and the severity of frailty (Table 4).

During the study period, 60 patients (31.1\%) died. The causes of death included cardiovascular disease (8 cases), cerebrovascular diseases ( 7 cases), peritonitis (10 cases), non-peritonitis infections (22 cases) and malignancy ( 5 cases). In addition, 8 patients were converted to long-term hemodialysis, 11 patients received kidney transplantation, and 4 patients were transferred to other dialysis centers. At 24 months, patient survival was $83.3 \%, 80.4 \%, 57.6 \%$, and $52.2 \%$ for patients with nil, mild, moderate, and severe frailty, respectively (log rank test, $\mathrm{p}<0.0001$ ) (Figure 2), while technique survival was $72.9 \%, 70.0 \%$, $57.6 \%$, and $49.5 \%(\mathrm{p}=0.007$ ) (Figure 3 ). After adjusting for potential confounding factors by multivariate Cox regression analysis, however, frailty was not an independent predictor of patient survival (adjusted HR 1.21, 95\% CI 0.94 to 1.54) or technique survival (adjusted HR $1.19,95 \%$ CI 0.97 to 1.47 ).

\section{Discussion}

In the present study, we showed that frailty was prevalent in Chinese PD population. Frailty is highly prevalent in elderly Chinese PD patients, but is not uncommon amongst younger ones. Most importantly, frail PD patients have a high risk of requiring hospitalization, and their hospital stay tend to be prolonged for each admission.

The definition and optimal method of identifying frailty remain controversial. The original Fried criteria [13] defined frailty as a phenotype by the presence of at least 3 out of the 5 components: unintentional weight loss, exhaustion, weakness, slow walking speed, and low physical activity. However, this approach involves the completion of multiple items in different questionnaires, as well as the measurement of walking speed and grip strength with appropriate instruments. Unfortunately, these assessments may not be easily accomplished in busy outpatient settings. For example, one of the studies reported approximately $25 \%$ of the recruited patients had missing data in at least one domain [14]. Moreover, cut-off points for physical performance tests may vary between different ethnic groups as well as in CKD patients. In the present study, we chose a simple but validated Chinese questionnaire that consists of 30 straightforward dichotomous questions to assess frailty [7]. Our approach 


\section{Kidney Blood Pressure Research}

may therefore simplify the procedure of assessment and facilitate the routine screening of frailty. In addition, our approach allows gradation of frailty, and the cumulative deficit model appears to be consistent with the concept of progressive accumulation of deficits in various physiological systems.

The prevalence of frailty in Chinese PD patient we found in this study appears to be higher than previous reports. Two studies recruited pre-dialysis CKD patients and determined frailty by modified Fried's criteria (which consisted of the original five components but assessed by different instruments) [5, 14]. The prevalence of frailty was reported to be 14$21 \%$, when frailty was defined by the presence of three or more of the five components [5, 14]. On the other hand, our results concurred with the finding of two large cohort studies, which reported that approximately $70 \%$ of dialysis populations were frail $[4,15]$. These two cohorts modified the original Fried's criteria by adopting larger proportions of selfreported criteria. The simplified version by Bao et al indeed omitted the domain of 'weight loss' but the estimated prevalence did not show substantial difference compared with another national cohort (73\% [15] versus $68 \%$ by Johansen et al [4]). Nevertheless, it is important to note that these previous studies revealed a considerable proportion of young dialysis patients being frail, which reinforced the notion that frailty was not confined to elderly patients.

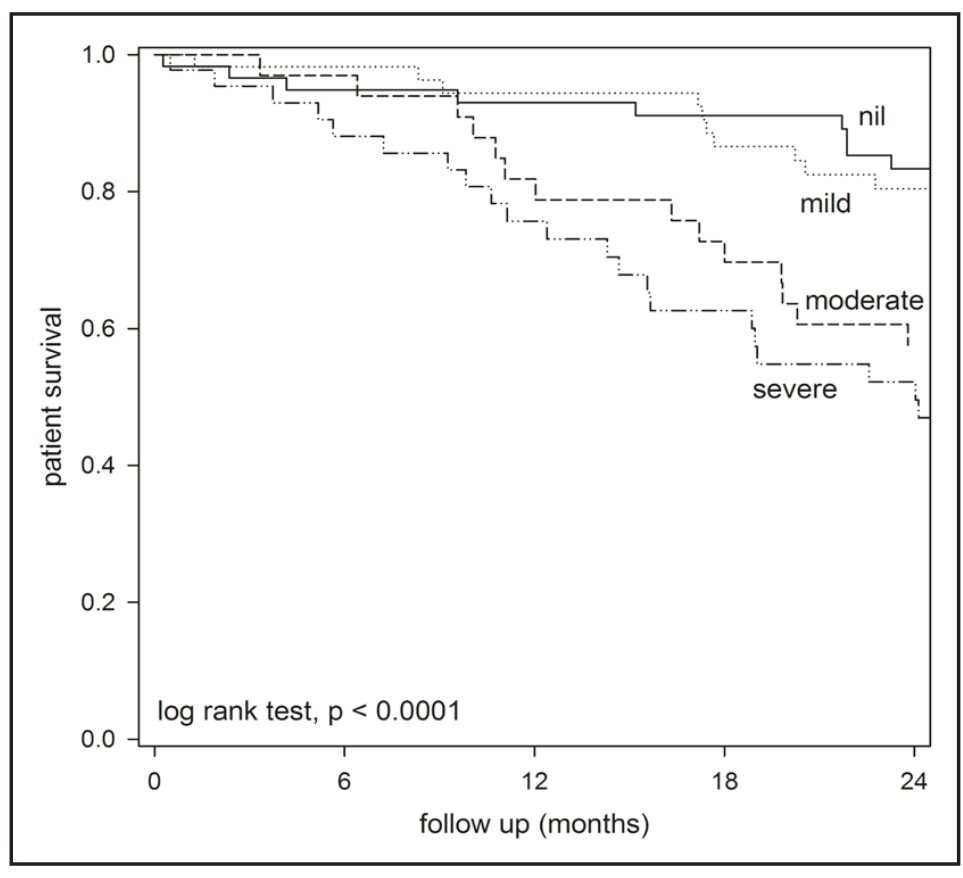

Fig. 2. Kaplan-Meier plot of patient survival. Patients were grouped according to the degree of frailty. Data are compared by the log rank test.

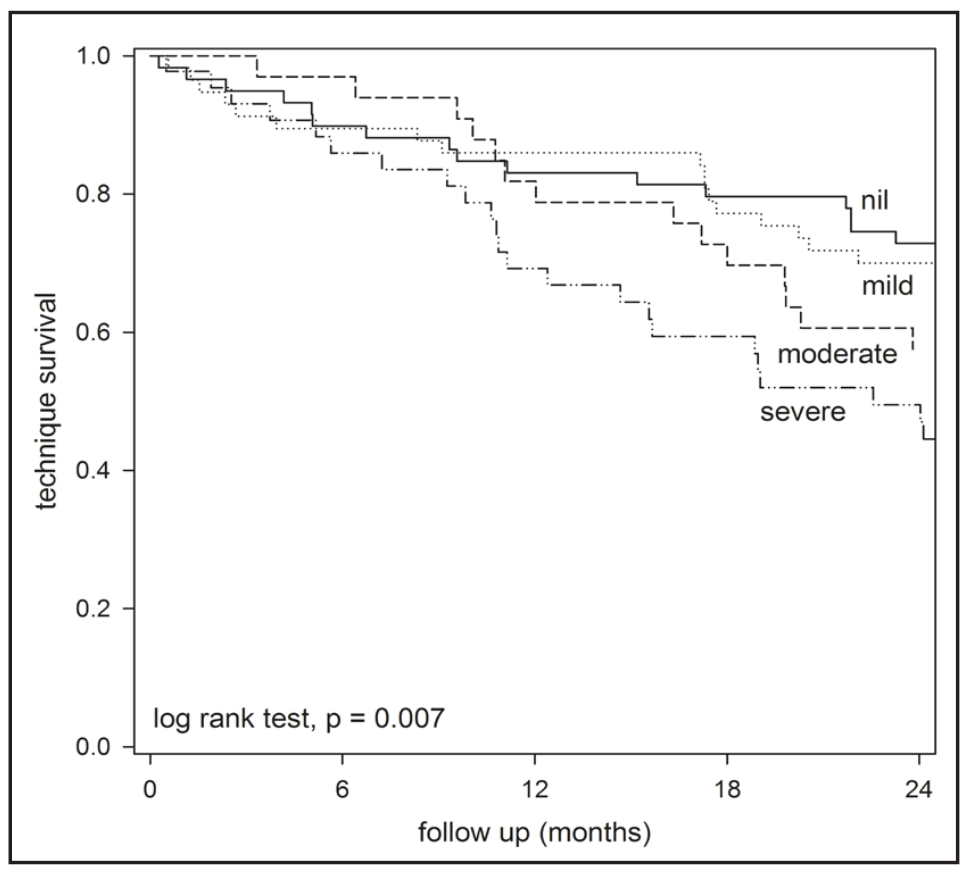

Fig. 3. Kaplan-Meier plot of technique survival. Patients were grouped according to the degree of frailty. Data are compared by the log rank test. 


\section{Kidney Blood Pressure Research}

In the present study, Frailly Score showed significant correlation with various clinical, anthropometric, biochemical and nutritional parameters. Amongst all parameters, Frailty Score had moderately strong correlation with serum albumin $(r=-0.33, p<0.0001)$, MIS ( $r=$ $0.59, \mathrm{p}<0.0001)$ and overall SGA score $(\mathrm{r}=-0.44, \mathrm{p}<0.0001)$. In fact, $\mathrm{SGA}$ was previously shown to be a reliable indicator of nutritional status in dialysis population, with good correlations with serum pre-albumin level and mid-arm circumference [16]. On the other hand, MIS correlated well with serum levels of inflammatory markers such as C-reactive protein (CRP) in ambulatory hemodialysis patients [9]. Our results suggested that malnutrition and systemic inflammation may be important contributing factors to development of frailty. Interestingly, Frailty Score was inversely correlated with weekly total $\mathrm{Kt} / \mathrm{V}(\mathrm{r}=-0.26, \mathrm{p}=$ $0.001)$ and residual GFR ( $r=-0.22, p=0.003)$. Although this association was weak to modest only, it was not described in previous studies. Sarcopenia is the one of the salient features of frailty. Patients who were underdialysed and/or losing their residual renal function may have enhanced oxidative stress, higher levels pro-inflammatory cytokines and uremic toxins. This may lead to protein catabolism and muscle breakdown, which eventually contribute to decrease in muscle strength and low activity [17]. However, whether increase in dialysis dose and preservation of residual renal function could modify the clinical course of frailty remain to be solved.

We found that Frailty Score is an independent factor associated with the duration of hospitalization. Notably, severely frail PD patients required twice as many hospital admission than patients who were not frail, and, for each admission, severely frail patients had to stay twice as long in the hospital. Since frail PD patients consume a disproportionately large amount of health care resource, routine assessment of frailty should be seriously considered in all PD patients.

In contrast to published evidences $[4,15]$, our study did not show a significant association between frailty and patient survival. This discrepancy may be related to the small sample size, different ethnicity and different dialysis modalities. Previous studies recruited more than a thousand of Caucasian patients on dialysis, and majority of them were on hemodialysis [4, 15]. Notably, Johansen's group suggested that hemodialysis, compared to peritoneal dialysis, was independently associated with frailty [4]. Whether such association would contribute to the difference in patient survival would require further studies. Another unexpected finding was that peritonitis rate was not significantly increased in frail PD patient. This is probably because of the higher prevalence of requiring assistance for PD amongst frail patients.

Our study had several limitations. First, the cross-sectional assessment of frailty did not address the progression of frailty in PD patients. It is possible that repeated measurement of Frailty Score may provide extra prognostic information or serve to monitor treatment response if interventions targeted on frailty have been initiated. Moreover, the cross-section, observational design of the present study did not allow us to establish causality but just associations between frailty and underlying comorbidities and nutritional status. Second, our assessment of frailty only included self-reported criteria. Our approach may theoretically lead to over-estimation of frailty because patients may report functional difficulty before their performance deteriorates to the level that meets the criteria by objective measures [18]. In fact, measured performance and self-reported physical function may capture two distinct stages of declining function [19]. Those who meet self-reported criteria only may represent an intermediate or pre-frail phenotype and allow early intervention to be initiated. Given that the data on hospitalization was convincing, a Kaplan-Meir curve showing the time to first hospitalization may be interesting. Unfortunately, the data were not available because of the limitations in our original study design. Third, previous studies suggested that the presence of peritoneal dialysate during body composition measurement may lead to overestimation of extracellular water (and thus hydration status), fat free mass and soft lean mass $[20,21]$. Therefore it may be ideal to measure body composition with an empty abdomen; yet this was not performed in our study due to logistic reasons and busy outpatient settings. Nevertheless, the degree of discrepancy may vary between the type of analyzer and the absolute magnitude of difference may be small [20]. Conflicting results were obtained when bioimpedance spectroscopy (in the present study) and multi-frequency bioimpedance 


\section{Kidney \\ Blood Pressure Research}

analysis were applied respectively [22]. More importantly, we have shown that change in body composition parameters (ovehydration and lean tissue mass) were significantly correlated with the degree of frailty as measured by the Frailty Score (Table 2). A previous study also reported that visceral fat content, as measured by bioimpedance analysis, is a simple method for predicting the risk of chronic kidney disease and metabolic syndrome in the general population [23]. Since the primary objective of our study was to determine the prevalence and contributing factors of frailty, we did not perform extensive analysis on the possible clinical implications of central obesity in PD patients, which has been examined in detail in another study by our group [24].

In spite of the shortcomings, our results do support the clinical relevance of frailty in Chinese PD patients. In our opinion, frailty should be screened and assessed regularly in dialysis patients. Current evidence suggests that frailty may be modifiable, although the treatment required a multifaceted approach [17]. Although intensive exercise training may not be feasible for the elderly population, gentle exercise programme has been shown to improve physical performance and reduced the fall risk in elderly with incipient frailty [25, 26]. Whether similar intervention would be beneficial in CKD and dialysis patients requires further studies.

\section{Conclusion}

Frailty was highly prevalent in Chinese PD patients. It may serve as a better surrogate than chronological age for the assessment of functional capacity. Frail PD patients were more likely to be hospitalized, and they required a longer stay for each hospital admission. Our study further supported the use of a self-reported Frailty Score questionnaire as a simple tool for screening of frailty in PD patients.

\section{Disclosure Statement}

The authors confirm that they do not have any conflict of interests and nothing to disclose.

\section{Acknowledgement}

This study was supported in part by the Chinese University of Hong Kong (CUHK) research account 6901031. The results presented in this paper have not been published previously. The authors declare no conflict of interest.

\section{Reference}

1 Fried LP, Ferrucci L, Darer J, Williamson JD, Anderson G: Untangling the concepts of disability, frailty, and comorbidity: Implications for improved targeting and care. J Gerontol A Biol Sci Med Sci 2004;59:255-263.

2 Clegg A, Young J, Iliffe S, Rikkert MO, Rockwood K: Frailty in elderly people. Lancet 2013;381:752-762.

3 Shlipak MG, Stehman-Breen C, Fried LF, Song X, Siscovick D, Fried LP, Psaty BM, Newman AB: The presence of frailty in elderly persons with chronic renal insufficiency. Am J Kidney Dis 2004;43:861-867.

4 Johansen KL, Chertow GM, Jin C, Kutner NG: Significance of frailty among dialysis patients. J Am Soc Nephrol 2007;18:2960-2967.

5 Wilhelm-Leen ER, Hall YN, Tamura MK, Chertow GM: Frailty and chronic kidney disease: the Third National Health and Nutrition Evaluation Survey. Am J Med 2009;122:664-671.

6 Beddhu S, Zeidel ML, Saul M, Seddon P, Samore MH, Stoddard GJ, Bruns FJ: The effects of comorbid conditions on the outcomes of patients undergoing peritoneal dialysis. Am J Med 2002;112:696-701. 


\section{Kidney \\ Blood Pressure Research}

7 Chan LS. Chronic disease self-management in Hong Kong Chinese older adults living in the community. [PhD thesis]. http://etheses.lib.cuhk.edu.hk/servlet/list?action=abstract_tab\&barcode $=920150488$

8 Enia G, Sicus C, Alati G, Zoccali C: Subjective global assessment of nutrition in dialysis patients. Nephrol Dial Transplant 1998;8:1094-1098.

9 Kalantar-Zadeh K, Kopple JD, Block G, Humphreys MH: A malnutrition-inflammation score is correlated with morbidity and mortality in maintenance hemodialysis patients. Am J Kidney Dis 2001;38:1251-1263.

10 Bergstrom J, Heimburger O, Lindholm B: Calculation of the protein equivalent of total nitrogen appearance from urea appearance. Which formulas should be used? Perit Dial Int 1998;18:467-473.

11 Forbes GB, Bruining GJ: Urinary creatinine excretion and lean body mass. Am J Clin Nutr 1976;29:13591366.

12 Kwan BC, Szeto CC, Chow KM, Law MC, Cheng MS, Leung CB, Pang WF, Kwong VW, Li PK: Bioimpedance spectroscopy for the detection of fluid overload in Chinese peritoneal dialysis patients. Perit Dial Int 2014;34:409-416.

13 Fried LP, Tangen CM, Walston J, Tangen CM, Walston J, Newman AB, Hirsch C, Gottdiener J, Seeman T, Tracy R, Kop WJ, Burke G, McBurnie MA: Frailty in older adults: evidence for a phenotype. J Gerontol A Biol Sci Med Sci 2001;56:M146-M156.

14 Roshanravan B, Khatri M, Robinson-Cohen C, Levin G, Patel KV, de Boer IH, Seliger S, Ruzinski J, Himmelfarb J, Kestenbaum B: A prospective study of frailty in nephrology-referred patients with CKD. Am J Kidney Dis 2012;60:912-921.

15 Bao Y, Dalrymple L, Chertow GM, Kaysen GA, Johansen KL: Frailty, dialysis initiation, and mortality in endstage renal disease. Arch Intern Med Jul 2012;172:1071-1077.

16 Visser R, Dekker FW, Boeschoten EW, Stevens P, Krediet RT: Reliability of the 7-point subjective global assessment scale in assessing nutritional status of dialysis patients. Adv Perit Dial 1999;15:222-225.

17 Kim JC, Kalantar-Zadeh K, Kopple JD: Frailty and Protein-Energy Wasting in elderly patients with end stage kidney disease. J Am Soc Nephrol 2013;24:337-351.

18 Johansen KL, Dalrymple LS, Delgado C, Kaysen GA, Kornak J, Grimes B, Chertow GM: Association between body composition and frailty among prevalent hemodialysis patients: a US Renal Data System special study. J Am Soc Nephrol 2014;25:381-389.

19 Johansen KL, Dalrymple LS, Delgado C, Kaysen GA, Kornak J, Grimes B, Chertow GM: Comparison of selfreport-based and physical performance-based frailty definitions among patients receiving maintenance hemodialysis. Am J Kidney Dis 2014;64:600-607.

20 Kang SH, Cho KH, Park JW, Yoon KW, Do JY: Body composition measurements using bioimpedance analysis in peritoneal dialysis patients are affected by the presence of dialysate. Nephrology (Carlton) 2014;19:727731.

21 Davenport A: Does peritoneal dialysate affect body composition assessments using multi-frequency bioimpedance in peritoneal dialysis patients? Eur J Clin Nutr 2013;67:223-225.

22 Parmentier SP, Schirutschke H, Schmitt B, Schewe J, Herbrig K, Pistrosch F, Passauer J: Influence of peritoneal dialysis solution on measurements of fluid status by bioimpedance spectroscopy. Int Urol Nephrol 2013;45:229-232.

23 Kang SH, Cho KH, Park JW, Yoon KW, Do JY: Association of visceral fat area with chronic kidney disease and metabolic syndrome risk in the general population: analysis using multi-frequency bioimpedance. Kidney Blood Press Res 2015;40:223-230.

24 Szeto CC, Kwan BC, Chow KM, Leung CB, Cheng MS, Law MC, Li PK: Metabolic syndrome in peritoneal dialysis patients: choice of diagnostic criteria and prognostic implications. Clin J Am Soc Nephrol 2014;9:779-787.

25 Faber MJ, Bosscher RJ, Chin A Paw MJ, van Wieringen PC: Effects of exercise programs on falls and mobility in frail and pre-frail older adults: A multicenter randomized controlled trial. Arch Phys Med Rehabil 2006;87:885-896.

26 Greco A, Paroni G, Seripa D, Addante F, Dagostino MP, Aucella F: Frailty, disability and physical exercise in the aging process and in chronic kidney disease. Kidney Blood Press Res 2014;39:164-168. 